\title{
Interdisciplinarité, interdisciplinarités (2)
}

Interdisciplinarity, interdisciplinarities (2)

\section{Béatrice Fleury et Jacques Walter}

\section{OpenEdition}

\section{Journals}

Édition électronique

URL : http://journals.openedition.org/questionsdecommunication/2643

DOI : 10.4000/questionsdecommunication.2643

ISSN : 2259-8901

\section{Éditeur}

Presses universitaires de Lorraine

\section{Édition imprimée}

Date de publication : 30 juin 2011

Pagination : 143-154

ISBN : 978-2-8143-0084-2

ISSN : 1633-5961

\section{Référence électronique}

Béatrice Fleury et Jacques Walter, «Interdisciplinarité, interdisciplinarités (2) », Questions de communication [En ligne], 19 | 2011, mis en ligne le 21 juin 2018, consulté le 19 avril 2019. URL : http:// journals.openedition.org/questionsdecommunication/2643; DOI : 10.4000/ questionsdecommunication.2643 


\section{$>$ ÉCHANGES}

BÉATRICE FLEURY

Centre de recherche sur les médiations

Université Nancy 2

Beatrice.Fleury@univ-nancy2.fr

JACQUES WALTER

Centre de recherche sur les médiations

Université Paul Verlaine-Metz

jacques.walter@univ-metz.fr

\section{INTERDISCIPLINARITÉ, INTERDISCIPLINARITÉS (2)}

Résumé. - Prolongeant le débat engagé dans la livraison précédente, Jérôme Bourdon, historien et chercheur en sciences de l'information et de la communication, Jean-Paul Resweber, philosophe, et Laurent Vidal, anthropologue, discutent le point de vue que défend Patrick Charaudeau (2010) sur l'interdisciplinarité. Deux idées principales largement étayées par chacun - ressortent des contributions : la science est le résultat d'une maturation des idées, méthodes et théories, ce qui conduit à formuler l'hypothèse selon laquelle l'interdisciplinarité est au fondement même de la naissance des disciplines; elle est aussi au cœur d'enjeux qui ne sont pas seulement scientifiques.

Mots clés. - Discipline, interdisciplinarité, pluridisciplinarité, transdisciplinarité, anthropologie, histoire, philosophie. 


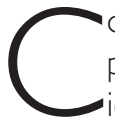
omme cela fut le cas lors des échanges publiés dans la livraison précédente de Questions de communication (2010), deux des chercheurs ici réunis s'expriment à la première personne, notamment lorsqu'ils exposent - situations concrètes à l'appui - des façons spécifiques de pratiquer l'interdisciplinarité. Par exemple, habitué des travaux conduits dans le domaine de la santé où peuvent être amenés à collaborer des chercheurs de disciplines diverses, l'anthropologue Laurent Vidal traite des attendus, profits et difficultés des recherches de cet ordre. Ainsi montre-t-il, notamment à partir d'enquêtes sur le sida conduites en Afrique, comment les chercheurs doivent composer, non seulement entre eux mais aussi avec la commande publique. Quant à Jérôme Bourdon, historien de formation, aujourd'hui chercheur en sciences de l'information et de la communication, il raconte une anecdote personnelle la soutenance de sa thèse - qui est pour lui l'occasion d'illustrer les écarts théoriques et méthodologiques pouvant distinguer des chercheurs travaillant sur un même objet, en l'espèce la radio-télévision française. On voit alors des identités disciplinaires se faire et se défaire au contact d'autres identités, en même temps que se constituent des domaines et préoccupations scientifiques. Tel est d'ailleurs l'angle par lequel le philosophe Jean-Paul Resweber approche l'interdisciplinarité, considérant que cette dernière est à la fois une étape assurant la transition entre la pluri- et de la transdisciplinarité, et un parcours ou se nouent et se dénouent des savoirs en même temps que s'unifient des idées, des approches, des notions et/ou des concepts.

Parmi les « familles » d'arguments développés, nous en retiendrons deux: celles ayant trait à ce qui concerne le cadre et les conditions de fabrication de la science; celles concernant la dimension politique dont peut être investie l'interdisciplinarité. De la sorte, nous verrons que, d'une part, l'interdisciplinarité est une donnée structurante des disciplines et que, d'autre part, l'invoquer ou la rendre « obligatoire » peut témoigner d'attendus et d'enjeux autres que ceux seulement relatifs à la connaissance.

\section{Une discipline se fabrique, une interdiscipline aussi}

Trois idées fortes ressortent des propos de Jean-Paul Resweber, Laurent Vidal et Jérôme Bourdon. Pour le premier, il n'y a pas de discipline sans interdiscipline; pour le second, il n'y pas de recherche sans interdiscipline; pour le dernier, « l'interdisciplinarité est partout, c'est-à-dire nulle part ». Si les points de vue se rejoignent, leur portée diffère : elle conduit le premier à revenir aux origines d'une discipline pour en suivre la construction; elle pousse le deuxième à exposer les conditions de mise en œuvre d'une recherche. Elle guide le troisième sur une voie décalée, celle d'une distance critique vis-à-vis de l'interdisciplinarité.

À l'instar de Michel Foucault (1966), Jean-Paul Resweber part de l'idée selon laquelle, au commencement, une discipline s'organise autour de savoirs 
empiriques « disons de savoir-faire qui, peu à peu, vont être soumis à un travail de formalisation. Ainsi mis en perspective, les savoirs se transmuent en connaissances ». Via la vulgarisation qu'en font les chercheurs, elles-mêmes se transmuent en savoir axiomatisé, ce qui fait qu'on passe « des pratiques à la théorisation pour en revenir à la pratique ». Dans ce mouvement, la discipline obéit donc «à une double logique d'extraction qui arrache les savoirs à la culture, et d'inculturation qui les reverse au compte de la culture. Par la médiation des connaissances qu'elle élabore, elle transforme les savoirs empiriques en savoirs susceptibles d'être partagés, une fois constitués en "capital symbolique" ». Mais le philosophe va plus loin. Toujours à l'instar de Michel Foucault (1969), il explique que « les sciences humaines se développent à partir de trois disciplinessouches : la biologie, l'économie et la linguistique ». Or, si les sciences humaines se constituent en empruntant à des disciplines-souches, elles le font aussi en annexant des « concepts appartenant à des disciplines déjà constituées ou en cours de constitution ». D'où l'idée selon laquelle les disciplines, comme les langues, s'éteindraient si elles ne créaient pas de nouveaux mots, concepts et/ou façons d'approcher le réel... Du coup, pour lui, pluri- inter- et intra-disciplinarités ne sont pas distinctes, mais représentent chacune l'une des étapes d'un même cheminement, l'interdisciplinarité constituant le milieu « au triple sens de ce terme : l'écart ou la marge, l'espacement ou l'intervalle et le juste milieu visé par l'interprétation ».

Pas de désaccord avec Patrick Charaudeau, mais un déplacement de l'approche que Jean-Paul Resweber qualifie lui-même comme étant en marge et en complément de celle du linguiste: « J'ai surtout insisté sur les finalités anthropologiques qui cristallisent les intérêts ou les enjeux de l'interdisciplinarité et sur la méthode herméneutique nécessaire à leur réalisation, sans pour autant négliger les marqueurs qui témoignent du croisement et de la récollection des savoirs $\gg$.

Ce déplacement conduisant à ne pas seulement envisager l'interdisciplinarité en termes de profits est aussi celui que, dans une certaine mesure, Laurent Vidal adopte. Pour lui, une réflexion sur l'interdisciplinarité doit « s'accompagner, ou à tout le moins se mettre en miroir, de la démarche réciproque qui consiste à penser la question de l'interdisciplinarité non pas tant à partir de "sa discipline" (Patrick Charaudeau le fait aussi), mais au travers d'un décryptage de la fabrique de sa discipline ». Pourquoi? Parce qu'un point aveugle demeure quand le geste de l'interdisciplinarité est prôné, celui qui permettrait d'identifier son porteur ou son inspirateur : « En demandant "qui fait cette interdisciplinarité?", j'attends - peutêtre un peu paradoxalement - moins une réponse formelle à la question qu'un retour sur la construction de la recherche impliquant plusieurs disciplines ». Pour attester de l'importance que, selon lui, il y aurait à poser la question, il revient sur les traces de deux études conduites en Afrique (Côte d'Ivoire, Sénégal) dans le domaine de la santé. L'une - « Santé maternelle » (200l-2005) - associait des anthropologues, des médecins et des spécialistes de la « mobilisation sociale et 
politique » autour d'un objectif de compréhension des pratiques des personnels de santé prenant en charge les accouchements difficiles, et d'intervention visant à les transformer; l'autre - «Évaluation » - faisait travailler ensemble anthropologues, économistes, démographe et épidémiologiste pour évaluer « les conditions de mise en œuvre d'une action lancée par une agence onusienne ONUSIDA - qui expérimentait en Côte d'Ivoire, entre 1999 et 200 I, la délivrance de traitements du $\mathrm{V} \mid \mathrm{H} \gg$. Dans les deux cas, Laurent Vidal était responsable ou co-responsable de l'étude. Aussi, tentant de répondre à la question « qui détermine l'interdisciplinarité? », il affirme qu'il ne s'agit aucunement de la personnalité assurant une fonction de direction. En outre, il démontre que le mélange des disciplines ne s'opère pas à partir de principes posés une fois pour toutes, mais dans un cadre en permanence négocié « et que c'est dans cette confrontation-là que naissent des espaces d'autonomie mais aussi de possibles blocages ». Or, on le sait, ces blocages peuvent subsumer le cadre disciplinaire. En conséquence, constatant de réelles connivences d'intérêts, d'approches, de vocabulaire entre chercheurs de secteurs différents, Laurent Vidal s'interroge: «Comment une telle proximité de centres d'intérêt peut-elle aller de pair avec de difficiles collaborations sur le "terrain"? ». Dans le cadre des études qu'il a conduites, il explique ceci par le fait que, d'un côté, on trouve des chercheurs, de l'autre, des opérateurs: «Des métiers donc différents, qui décident de participer à un même projet mais qui, lorsqu'ils doivent traiter des questions dont ils s'estiment les spécialistes, tendent à vouloir affirmer leur expérience, leur savoir-faire $\gg$.

À l'heure du vivre et travailler ensemble à l'université, ces propos font ô combien écho à des situations maintes fois rencontrées. On y perçoit la manifestation du besoin de légitimation de soi qui passe - est-il besoin de le dire : parfois, souvent? - par le refus de l'autre ou le déni de ce qui singularise celui-ci. À cet égard, une phrase, adressée récemment dans une réunion aux auteurs de cette introduction, est emblématique: «Mais comment pouvons-nous travailler avec vous à un tel programme? Vos problématiques ont trente à quarante ans de retard! ». Prononcée par le directeur (ayant d'ailleurs une haute idée de lui-même) d'un laboratoire hébergeant des chercheurs appartenant à une discipline reconnue de longue date, cette phrase était destinée à sonner le glas d'une collaboration - imposée par des présidents d'universités - entre ces chercheurs et ceux issus d'une discipline plus récente, en l'occurrence les sciences de l'information et de la communication (SIC). Si méprisant et faux soit-il, le propos exprime clairement ce que beaucoup pensent tout bas : les disciplines ne se valent pas. Elles sont régies par une échelle de grandeur - à peine masquée - au sommet de laquelle peuvent se situer les plus anciennes d'entre elles, comme la philosophie.

À ces propos et à la conclusion que nous en tirons, Jérôme Bourdon rétorquerait probablement qu'opposer une discipline à une autre est sans objet, la notion même de discipline pouvant se révéler hasardeuse. Au demeurant, en préambule à sa contribution, il écrit: «Avant de croiser les disciplines, il faut savoir où 
nous en sommes de la question disciplinaire, sinon, nous ressemblerons fort à la gentry anglaise chassant le renard, décrite par Oscar Wilde : I'innommable (la discipline) chassant l'immangeable (l'interdisciplinarité) ». C'est donc ce à quoi il s'emploie. Convoquant Roger Chartier (1998), il pose l'idée selon laquelle concept et territoire sont deux des éléments de définition pouvant être mis à contribution pour aider à comprendre les disciplines. Ainsi précise-t-il que certaines se reconnaissent plus dans l'un que dans l'autre. Et de développer une argumentation serrée de la façon dont les unes ou les autres investissent des territoires et/ou des concepts spécifiques mais s'y affrontent aussi. II explique:

«L'axe qui va du primat de la conceptualisation au primat de la description des objets est certainement le plus fécond pour comprendre les oppositions entre disciplines. À ce jeu, on est toujours le philosophe de quelqu'un (trop abstrait, votre affaire, vous ignorez les faits), ou son géographe (trop concrète, et donc faussement concrète, cher ami, votre description, vous oubliez que le réel est construit - même si je suis très content, quand même, de piocher dans votre description) ».

Et si l'on y ajoute la question et les débats concernant les méthodes, on a, en substance, quelques-unes des difficultés majeures pouvant empêcher qu'un dialogue ne s'établisse entre des chercheurs de disciplines différentes. Mais, Laurent Vidal complète, et on le suit totalement: « Les échanges, les éventuelles divergences, ne se réduisent pas à des confrontations disciplinaires, même si elles peuvent parfois y renvoyer. Ce sont aussi des traditions de recherche ou d'action qui se rencontrent, des conceptions de l'action elle-même qui sont en discussion et, enfin, des approches différentes du travail ». On pourrait ajouter : ce sont aussi des individualités et un regard que l'on porte sur soi-même et les autres...

Enfin, dans sa quête d'une définition de ce qu'est une discipline et doutant que les contours de celle-ci puissent connaître une forme de stabilisation, Jérôme Bourdon évoque la «stratégie de reconversion de ressources d'un champ universitaire dans un autre ». Ainsi mentionne-t-il « la reconversion des littéraires dans la sociologie des médias, dans les études culturelles, dans l'analyse du discours, qui permet de sauver au moins une partie d'un capital culturel chèrement acquis ». $\|$ en vient aussi à parler de ces voyages qui conduisent une discipline à en utiliser une autre - à en faire une science auxiliaire -, telle la sémiologie qui aurait acquis un statut « de science auxiliaire d'analyse des messages (singulièrement de l'image), ce qui est une défaite par rapport à l'ambition initiale ».

L'analyse de Jérôme Bourdon est donc à distance du regard encourageant que Patrick Charaudeau portait sur ces voyages. En les envisageant sous l'angle d'une mise en contexte, Jérôme Bourdon met à nu les conflits dont ils peuvent être une résultante. L'interdisciplinarité oui, mais à condition de poser la question du sens qu'elle recouvre... Or, du sens aux conditions d'exercice de celle-ci, il n'y a qu'un pas. Par exemple celui qui permet d'interroger les enjeux - politiques par exemple - de l'interdisciplinarité. 


\section{Et si l'on parlait des enjeux que recouvre I'interdisciplinarité?}

Parmi les enjeux dont l'interdisciplinarité est une résultante, se trouve la question du pouvoir. Figurant en bonne place dans toutes les contributions, elle ne fait pas pour autant l'objet d'un traitement analogue dans chacune. Jean-Paul Resweber fait ce constat: « Les pouvoirs obligent les savoirs à se plier à d'autres enjeux que ceux dont ils se sont dès le départ dotés, à plier les intérêts particuliers à des intérêts plus généraux ». En revanche, pour Laurent Vidal, « les données du système, le "cadre" de l'action (la demande se présente ainsi; les responsabilités dans le projet sont les suivantes) ne suffisent pas à guider l'intervention de l'individu, du chercheur et [... ], naturellement, à l'inverse, celui-ci n'est pas non plus maître de son action - sous prétexte qu'il serait anthropologue ou responsable d'une équipe ». Un regard contrasté donc pour une question d'importance qui conduit Jérôme Bourdon à s'engager dans une autre voie, celle d'une petite interdisciplinarité, ou plus modestement, d'interméthodologie.

C'est dans la démonstration de Jean-Paul Resweber que la question des enjeux disciplinaires/interdisciplinaires est la plus fortement posée, lui qui expose l'idée selon laquelle « l'herméneutique interdisciplinaire s'inscrit dans une visée anthropologique ». Pour en expliciter et fonder certains traits, il croise et fait se compléter les analyses de Jacques Lacan (1962-1963, 1966, 1975-1976), Jürgen Habermas (1969, 1982) et Paul Ricœur (1990, 2005). Plus précisément, la référence au premier permet d'envisager la part fondamentale de la quête de vérité dans le savoir. Mais cette quête est particulière. Conçue comme sens absent, elle est impossible à thématiser et à ériger en axiome :

« Voilà bien pourquoi elle peut faire "bouger" et "s'entrechoquer" les savoirs, afin qu'ils se structurent en un savoir - que Jacques Lacan appelle aussi la langue -, savoir capable de tenir lieu du réel dont il est la longue et patiente écriture. [...] En provoquant le conflit des savoirs, elle [la vérité] fait le lit de la vérité qui exprime le désir de l'homme et contribue, ni plus ni moins, à reconfigurer le réel, celui qui est l'objet de la passion de chacun d'entre nous ».

En revanche, pour Jürgen Habermas, qui soumet les savoirs à une herméneutique critique, l'enjeu des savoirs n'est pas la vérité mais l'émancipation de l'homme, c'est-à-dire celle qui consiste à « se débarrasser des illusions et des idéologies, pour faire la vérité sur soi et sur les autres ». À ce sujet, Jean-Paul Resweber rappelle que Jürgen Habermas substitue à la notion de discipline celle de « rationalité », entendue au sens où « chaque discipline se définit moins par l'arsenal convenu du champ, du principe ou du paradigme, du cadre et des concepts, que par les logiques rationnelles qu'elles mettent en œuvre et par les intérêts que ces logiques mobilisent ». Ainsi voit-on des rationalités multiples (communicationnelles, discursives, rhétoriques, philosophiques, mathématiques, politiques et/ou artistiques) s'exprimer et dont le croisement compose une interdisciplinarité qui a pour finalité de «créer un milieu où elles peuvent être 
confrontées entre elles et soumises à une interprétation qui les redéfinit en fonction des intérêts humains ».

Et Paul Ricœur? Si ce dernier reprend le projet de Jürgen Habermas,

« c'est à la philosophie qu'il confie la fonction d'unification des disciplines. D'une façon plus précise, il voit dans l'herméneutique le lieu de l'articulation des méthodes et, par conséquent, celui de la résolution du conflit des méthodes ». En outre, « quelles que soient ses conclusions plus ou moins abouties, l'herméneutique vise à dé-substantialiser les catégories, les concepts et les représentations, en les situant, non sans les remanier, dans le projet ouvert d'une anthropologie dont la clef de voûte est la notion de subjectivité et d'intersubjectivité ».

Ceci posé, c'est aux lieux où l'interdisciplinarité se manifeste que Jean-Paul Resweber s'intéresse. Ceux-ci sont divers et couvrent nombre de domaines de l'échange, à commencer par le langage. On y trouve les médias, l'enseignement, la recherche, l'expertise, la littérature... Dans tous ces lieux, et sans que pour autant le philosophe la pose de la sorte, la question du pouvoir est à l'œuvre. Du reste, pour Jean-Paul Resweber, cette dimension - pourtant centrale - n'est qu'effleurée par Patrick Charaudeau; ce qui manque à la démonstration qu'il a mise en partage. Aussi le philosophe liste-t-il nombre de points relatifs à celle-ci :

« Le champ disciplinaire est labouré par les pouvoirs scientifiques, économiques et politiques. C'est un ensemble de pouvoirs qui fait la discipline : sociétés de discours, découpage des thèmes et des problèmes, sacralisation des références et culte des grands auteurs, incursion du politique et de l'économie dans la définition des programmes, dans la planification de la recherche, dans l'agrément des laboratoires de recherche, dans la reconnaissance et l'habilitation des diplômes, dans la valorisation professionnelle de certaines disciplines au détriment d'autres et surtout, de nos jours, dans l'institution de normes d'évaluation qui se justifient moins par l'efficacité qu'elles garantissent que par la rhétorique de la performance qui les légitime ».

Ces marques du pouvoir, on les connait; elles font partie du quotidien de la recherche et cadrent les pratiques scientifiques. Jean-Paul Resweber explique même qu'elles sont présentes dans les injonctions à l'interdisciplinarité qui « proviennent le plus souvent des pouvoirs administratifs et économiques, relayés par les pouvoirs politiques ». Effectivement, quels sont aujourd'hui les commanditaires de programmes de recherche qui oseraient faire l'impasse sur le postulat d'interdisciplinarité? On ne peut qu'en faire le constat : cette obligation participe de l'ordinaire de la recherche. Si bien que, par un effet boomerang dû au caractère systématisant de la pratique, elle peut aussi conduire à une forme de « disciplinarisation »... de l'interdisciplinarité. Un risque que perçoit Laurent Vidal et contre lequel il propose d'adopter la position défendue par Jacques Hamel (2005: III) qui envisage l'interdisciplinarité comme « un mariage de raison qui renvoie à l'intérêt et à la volonté de considérer les connaissances issues d'autres disciplines dans l'intention de mieux cerner et de mieux éclairer son objet propre et, de ce fait, d'en produire une connaissance plus précise ».

En effet, selon Laurent Vidal, il est important de « garder son identité », un conseil qu'il formule dans la partie conclusive de son propos et qui le conduit, 
à l'instar de Sylvie Fainzang (2003), à plaider « non pas tant pour la pluri- ou l'interdisciplinarité que pour ce qu'elle nomme la "co-disciplinarité" ». Toutefois, pour que la démarche puisse être réalisable, deux conditions sont à respecter: faire en sorte que l'éloignement entre disciplines soit mesuré, la distance pouvant empêcher la compréhension des réflexes disciplinaires des autres chercheurs; encourager des moments d'explication pour lever toute mésinterprétation, lever des préventions ou a priori. L'anthropologue en livre un exemple issu de sa pratique :

« Face à ces acteurs de la santé, l'anthropologue doit tout d'abord expliquer sa démarche. Les questions qui mobilisent son attention peuvent intriguer : je mentionnerai l'étonnement de certains professionnels de santé qui attendent de l'anthropologue plus une étude sur les recours à la médecine traditionnelle ou les représentations de la maladie de leurs patients qu'une étude portant sur leurs pratiques à eux, soignants ».

Et si tout cela n'était qu'artifice ou affaire de circonstances? C'est ce que suggère Jérôme Bourdon lorsqu'il émet l'idée selon laquelle « les dynamiques sociales, institutionnelles (et personnelles, de surcroît [...]) nous forcent à des affiliations disciplinaires qui peuvent être vécues comme profondes, mais qui demeurent largement artificielles ». D'après ce chercheur, la promotion de l'interdisciplinarité peut « participer [...] d'un effort commun pour créer un rapport de forces favorables à l'émergence d'un objet, parfois le temps de l'obtention d'un contrat de recherche ou d'édition, parfois un peu plus durablement ». Une façon d'envisager l'interdisciplinarité qui correspond à l'idée qu'il avançait en préambule à sa démonstration et qui consistait à poser comme improbable une définition claire et stabilisée de la notion de discipline. En dépit de cette réserve, Jérôme Bourdon se risque pourtant à avancer des propositions. Une discipline serait structurée selon des caractères conceptuels, territoriaux et méthodologiques, d'une part, matériels et sociaux, d'autre part, individuels et psychologiques enfin, c'est-à-dire en lien avec « un attachement primitif qui seul donne du plaisir (le discours m'importe, la statistique m'importe, l'inconscient m'importe) qui travaille des individus et crée des attachements durables ».

Malgré tout, si ce chercheur parvient à dessiner à grands traits ce que serait une discipline, il peine à identifier les caractères les plus saillants de l'interdiscipline. Aussi se veut-il provocateur: «Cherchons [...] à prendre l'interdisciplinarité par un autre bout. Malgré le scepticisme de l'auteur, n'a-t-elle pas connu des réussites éclatantes? »». Et de regarder du côté de hautes figures dont les travaux sont fondés sur des rencontres entre disciplines comme Michel Foucault, Claude Lévi-Strauss ou Roland Barthes... pour aboutir à une impasse. Par exemple, concernant Michel Foucault, Jérôme Bourdon considère qu'il est ironique de le qualifier d'interdisciplinaire, lui qui transcende les disciplines (en l'occurrence la philosophie et l'histoire) plus qu'il ne les croise. Impasse aussi du côté de chercheurs comme Paul Ricœur ou Roger Chartier qui convoquent nombre de disciplines tout en revenant à la leur, la philosophie ou l'histoire. 
D'où un changement de cap avec cette question : « À défaut d'une "grande" définition de l'interdisciplinarité, qui suppose une "grande" définition des disciplines qui se rencontreraient et se fertiliseraient mutuellement, peut-on se rabattre sur une définition modeste? ». Là encore, il explique qu'il tient cette conception pour illusoire et s'en explique en convoquant un exemple qui lui est familier : la télévision. Revenons sur quelques points de sa démonstration. Jérôme Bourdon explique que, dans un cadre collaboratif autour de cet objet, l'historien procèdera à une analyse à large portée des archives concernant les hommes, les institutions, les programmes, les courriers, la critique... Le sémiologue s'attachera aux contenus... Le sociologue procédera à des interviews pour comprendre un fonctionnement, des règles, des usages... Le bilan est sans appel. Le profit tiré de cette collaboration est insatisfaisant:

« Je crains d'abord que les dialogues ne soient très limités, à nouveau. [...] II y a des dialogues possibles, mais ils sont fragiles et très inégalement répartis. La sémiologie dure est condamnée, par une langue spécifique, à s'isoler (ce qui fait aussi, à un autre point de vue, sa force). Le sociologue et I'historien dialogueront peut-être plus facilement, mais très vite ils rencontreront, eux aussi, leurs limites. Plus profondément, on retrouvera là les problèmes de l'intra-disciplinarité qui sont, aujourd'hui, tout aussi cruciaux, et montrent la tendance des sciences sociales à se diviser à l'infini, à l'abri de pavillons officiels qui abritent des marchandises de plus en plus diverses »».

\section{Conclusion}

Pour Jérôme Bourdon - qui titre sa contribution « L'interdisciplinarité n'existe pas »-, le mélange entre disciplines est l'affaire de chacun sans que pour autant il soit nécessaire, comme le prône Patrick Charaudeau de passer par un plaidoyer en sa faveur. Ainsi donne-t-il l'exemple des travaux d'Annette Kuhn (2008) qui ont trait aux « souvenirs de spectateurs de cinéma anglais d'avant-guerre pour comprendre la place du cinéma dans la vie quotidienne de l'époque ».Partant de là, il démontre que la combinaison de perspectives - historiennes et sociologiques adoptée par la chercheuse n'équivaut pas à une démarche interdisciplinaire. C'est « de la bonne histoire et de la bonne sociologie qui pratiquent tout simplement une diversité de méthodes, plus précisément de deux méthodes seulement, mais combinées de façon cohérente et systématique ». En cela, la posture critique de Jérôme Bourdon est proche de celle de ses deux collègues qui, sans récuser la position de Patrick Charaudeau, mettent néanmoins à distance les postulats que ce dernier défend. Pour Jean-Paul Resweber, la mise à distance résulte d'une prise en compte des enjeux épistémologiques de l'interdisciplinarité dont il a montré qu'ils ne sont pas seulement théoriques. Pour Laurent Vidal, elle est le fruit d'un exposé réflexif sur le « faire » disciplinaire, ici l'anthropologie.

Finalement, une question manque aux «Échanges» publiés dans les deux livraisons de Questions de communication, celle abordant l'ailleurs géographique des recherches dans le secteur de l'information-communication. Or, un dossier publié dans la revue canadienne Revue internationale. Communication sociale et 
publique vient à point nommé pour combler ce déficit, principalement deux contributions qui traitent des rapports entre communication et discipline dans les pays anglo-saxons. La première, rédigée par Oumar Kane, professeur au département de communication sociale et publique de l'université du Québec à Montréal, expose les attendus de la disciplinarisation de la communication, qu'il décrit comme étant une quasi-discipline.

«Malgré son inscription dans une discipline différente (la science politique), l'œuvre fondatrice d'Harold Innis a pu orienter une certaine forme d'institutionnalisation de la communication comme champ d'étude au Canada davantage qu'aux États-Unis. Ce processus a suivi des voies notablement différentes pour ce qui est des sciences de l'information et de la communication (SIC) françaises. Alors qu'en France l'institutionnalisation sociale a précédé et tiré rétrospectivement l'institutionnalisation épistémique (Boure, 2005), aux États-Unis la proximité de la discipline avec la rhétorique et la culture institutionnelle américaine expliquent la position centrale des départements plutôt que de l'État (Winkin, 2005) dans la structuration disciplinaire » (Kane, $2010: 88)$.

La comparaison entre la situation de la communication en France et celle dans les pays anglo-saxons est éclairante : elle montre la singularité du cas hexagonal et prouve l'ancrage culturel du débat ici initié. En effet, si l'interdisciplinarité est au fondement du caractère identitaire des sIC en France, en Amérique du Nord, le domaine est désigné comme étant un champ de recherche. Quoi qu'il en soit, l'institutionnalisation de ce champ n'est pas sans poser problème, ce que souligne aussi François Cooren, professeur à l'université de Montréal. Ainsi annonce-t-il :

« Les études en communication, c'est ma conviction, devraient à la fois se concentrer sur des objets communicationnels (le ou les foyer(s), donc) tout en développant des approches originales (des prismes, donc) qui puissent les singulariser en regard d'autres disciplines plus établies, comme l'anthropologie, la sociologie ou la psychologie » (Cooren, 2010 : I03- I 04).

Faisant largement référence à un article fondateur sur ces questions (Craig, 2009), François Cooren enjoint à unifier autant les travaux se reconnaissant sous le mot pavillon de communication que les chercheurs concernés. Et là encore, à l'image de ce que les chercheurs français peuvent connaître, des tensions peuvent se faire jour; elles sont autant internes (au sein d'un groupe particulier) qu'externes, la question de l'ancienneté de certaines disciplines pouvant se manifester:

« II faut donc - et, d'une certaine manière, la spécificité de notre discipline en dépendrait - partir des phénomènes communicationnels pour comprendre et expliquer le monde dans lequel nous vivons (que ce monde soit appréhendé dans ses dimensions psychologiques, sociales, économiques, politiques, voire même biologiques), au risque sinon de se faire phagocyter par d'autres disciplines historiquement et institutionnellement plus établies » (ibid. : 105).

Quelle leçon tirer de cette brève mise en regard? En France, cela fait désormais une quarantaine d'années que les interrogations actuellement à l'ordre du jour en Amérique du Nord se sont exprimées et ont donné forme à la discipline que sont les sic (Boure, 2006, 2007,2008). Pour autant, peut-on dire que les recherches conduites en France à l'aune de cette discipline sont unifiées? Indéniablement, non, chaque sous-champ de celle-ci pouvant lui-même se présenter selon des traits proches de ce qui constitue une discipline. Et le fait d'invoquer régulièrement 
l'interdisciplinarité comme élément d'unification n'est pas à même d'y parvenir. Ainsi l'interdisciplinarité ne peut-elle se contenter de fonctionner comme un slogan dont des esprits chagrins pourraient suspecter qu'il ne soit prononcé que pour masquer une certaine vacuité conceptuelle et/ou une forme de bricolage méthodologique. Un défi à relever... internationalement?

\section{Références}

Boure R., 2006, «L'histoire des sciences de l'information et de la communication (I ). Entre gratuité et réflexivité », Questions de communication, 10, pp. 277-295.

— 2007, « L'histoire des sciences de l'information et de la communication (2). Le cas des origines littéraires des SIC », Questions de communication, I I, pp. 257-287.

— 2008, « L'histoire des sciences de l'information et de la communication (3). Postures, concepts et méthodes en débat », Questions de communication, 13, pp. | 3 | - 152.

Charaudeau P., 2010, «Pour une interdisciplinarité "focalisée" dans les sciences humaines et sociales », Questions de communication, 17, pp. 195-222.

Chartier R., 1998, Au bord de la falaise. L'histoire entre certitudes et inquiétude, Paris, A. Michel.

Cooren Fr., 2010, « Le cahier des charges d'un (méta-) modèle constitutif de la communication: une proposition », Revue internationale. Communication sociale et publique, 3-4, pp. 103-122.

Craig R. T., 1999, «Communication theory as a field», Communication Theory, 9(2), pp. $119-161$.

Fainzang S., 2003, « Anthropologie et médecine : réflexions épistémologiques sur la co disciplinarité dans la recherche », La co-disciplinarité, Colloque de Lausanne, Institut universitaire d'histoire de la médecine et de la santé/ Institut d'anthropologie (Faculté des sciences sociales/Hautes écoles sociales). Accès : http ://halshs.archives-ouvertes.fr/ docs/00/I 0/52/86/PDF/Fainzang_-_anthropologie_medecine-_reflex_epistemol_sur_ co-disciplinarite.pdf.

Foucault M., 1966, Les mots et les choses, Paris, Gallimard.

- 1969, L'archéologie du savoir, Paris, Gallimard.

Habermas J., 1969, Connaissance et intérêt, trad. de l'allemand par G. Clémençon, Paris, Gallimard, 1976.

— 1982, Théorie de l'agir communicationnel, trad. de l'allemand par J.-M. Ferry, Paris, Fayard, 1987.

Hamel J., 2005, « Sociologie et interdisciplinarité, un mariage de raison? », A contrario, 3, I, pp. 107-115.

Kane $0 ., 2010$, « Institution et légitimation d'une quasi-discipline : le triple destin (sciences, études et champ) de la communication », 3-4, Revue internationale. Communication sociale et publique, 3-4, pp. 87-102.

Kuhn A., 2008, An Everyday Magic. Cinema and Cultural Memory, London, IB Tauris. 
Lacan J., 1962-1963, Séminaire, IX, Paris, Éd. Le Seuil, 2004.

— 1966, Écrits, T. 2, Paris, Éd. Le Seuil.

— 1975-1976, Séminaire, XXIII, Paris, Éd. Le Seuil, 2005.

Ricœur P., 1990, Soi-même comme un autre, Paris, Éd. Le Seuil.

- 2005, L'homme capable, texte écrit à l'occasion de la réception du Kluge Prize, Bibliothèque du Congrès USA. 